\title{
Case Report: Malignant Transformation of Noma: Repair by Forearm Flap
}

\author{
Xiang Ding, ${ }^{1} \dagger$ Qian-qian Wang, ${ }^{2} \dagger$ Yang Zhou, ${ }^{3}$ and Jin-cheng $X u^{2 *}$ \\ ${ }^{1}$ Department of Stomatology, The Second Affiliated Hospital of Bengbu Medical College, Bengbu, Anhui Province, China; ${ }^{2}$ Department of \\ Stomatology, The First Affiliated Hospital of Bengbu Medical College, Bengbu, Anhui Province, China; ${ }^{3}$ Editorial Department of Journal of Bengbu \\ Medical College, Bengbu, Anhui Province, China
}

\begin{abstract}
Noma is a progressive infectious disease manifested by a necrotic ulcer of the mouth and face. It usually occurs in poor, malnourished children, with about 30,000-140,000 cases each year and a low survival rate. The exact cause of noma remains unclear, but bacterial infection has been postulated to be a major cause of this disease. Antibiotics and improved nutrition could help inhibit the progression of noma, but most patients still require oral surgery because of the bacterial infection-induced tissue damage. In this study, we report an unusual case of a noma patient whose facial lesion developed a malignancy. The necrotic tissue and infectious area were surgically resected, and a forearm flap was used to repair the patient's facial defect. This case aimed at increasing people's awareness of tropical diseases such as noma.
\end{abstract}

\section{INTRODUCTION}

Noma, also known as cancrum oris, is a progressive infectious disease that results in tissue damage in the mouth and face with a high mortality and disability rate. Noma is mainly found among infants and young children from lowincome countries with poor hygiene environments; it rarely occurs in high-income countries. ${ }^{1}$ Noma has been found in soldiers with poor oral hygiene in the trenches before the founding of new China. ${ }^{2-4}$ According to statistics from the Geneva Study Group on Noma in 2017, there are an estimated $30,000-40,000$ cases per year worldwide. ${ }^{5}$ Bacterial infection is the main cause of the development of noma, and additional factors such as malnutrition, poor oral hygiene, and immunodeficiency have also been identified. ${ }^{6}$ The disease often progresses rapidly, and the bacterial infection results in tissue and bone damage. Therefore, facial surgery is necessary in most patients. In this study, we report a 66-year-old woman with noma sequelae that had progressed to cancer. We performed surgery and successfully removed infectious and necrotic tumor tissue from the patient.

\section{CASE DATA}

A 66-year-old woman who had left facial tissue defect with necrosis for more than 50 years was admitted to the Department of Stomatology of the First Affiliated Hospital of Bengbu Medical College. She suffered from measles infection 50 years ago that resulted in an oral mucosa rupture in her face, causing ulcers of the left maxillary tissue with severe chronic pain. The woman had applied some Chinese traditional herbs locally to the damaged area at first, but there was no improvement in pain or facial damage. In addition, the local health agency did not give her other suitable medical treatment in the early stage of the disease. This led to the development of left maxillary and facial tissue defects after several months. The patient subsequently did not receive proper treatment because of limited personal funds and local medical

\footnotetext{
*Address correspondence to Jin-cheng Xu, Department of Stomatology, The First Affiliated Hospital of Bengbu Medical College, No. 287, Changhuai Road, Longzihu District, Bengbu, Anhui Province, China. E-mail:w18895637183@163.com

$\dagger$ These authors contributed equally to this work.
}

capacity. During the long period of noma sequelae, this woman suffered from severe chronic pain and enormous psychological burden caused by the disease. About 1 month before the patient came to our hospital, she felt her left maxillary teeth loosen and removed the loose teeth by herself. However, there was continuous swelling and pain in the extraction fossa after that. A week later, there was no improvement in the symptoms of the left maxillary lesion. To seek treatment, the patient and her family members came to our hospital. On the first day of the patient's admission, we conducted a special examination on her: one-third of the face was deformed with left lower eyelid droop, left conjunctiva congestion, and incomplete wound closure. Tissue defects could be seen in the left maxillary and facial region, from the upper border to the lower eyelid, from the lower border to the left lower lip, red lips, and left corner of the mouth. The left upper lip was completely destroyed, from the right to the nasal septum; the nasal septum was exposed from the left to the upper edge of the cleft of the mouth. The surface of the defect revealed grayish yellow tissue with surrounding redness and swelling and a small amount of purulence. This limited her mouth opening to about $1 \mathrm{~cm}$, and the opening type was normal (Figure 1). The final clinical diagnosis was left facial malformation secondary to noma.

Operation steps are given as follows: 1 ) extended resection of the left maxillary focus and extraction of teeth 21, 22, 25, 33, and $34 ; 2$ ) left forearm flap free transplantation and microvascular anastomosis and left abdominal skin graft; and the postoperative repair of this patient after surgery, as shown in Figure 2.

There were no postoperative complications in the patient, and no vascular crisis occurred in the transplanted skin flaps. However, about 1 week after the operation, the flap near the nasal septum and lower eyelid wound edge ruptured with no ulceration and infection. After that, the left nasal cavity was exposed and the left eyelid cleft was incompletely closed. Pathological analysis revealed highly differentiated squamous cell carcinoma of the face. After discharge, the patient underwent monthly chemotherapy (pingyangmycin combined with the cyclophosphamide regimen) for 6 months, and there was no sign of recurrence and tumor metastasis after a year of follow-up (Figure 3). Because of the failure of the skin flap to repair the facial defect, we suggested that the patient should use a maxillofacial prosthesis to repair the facial defect after 


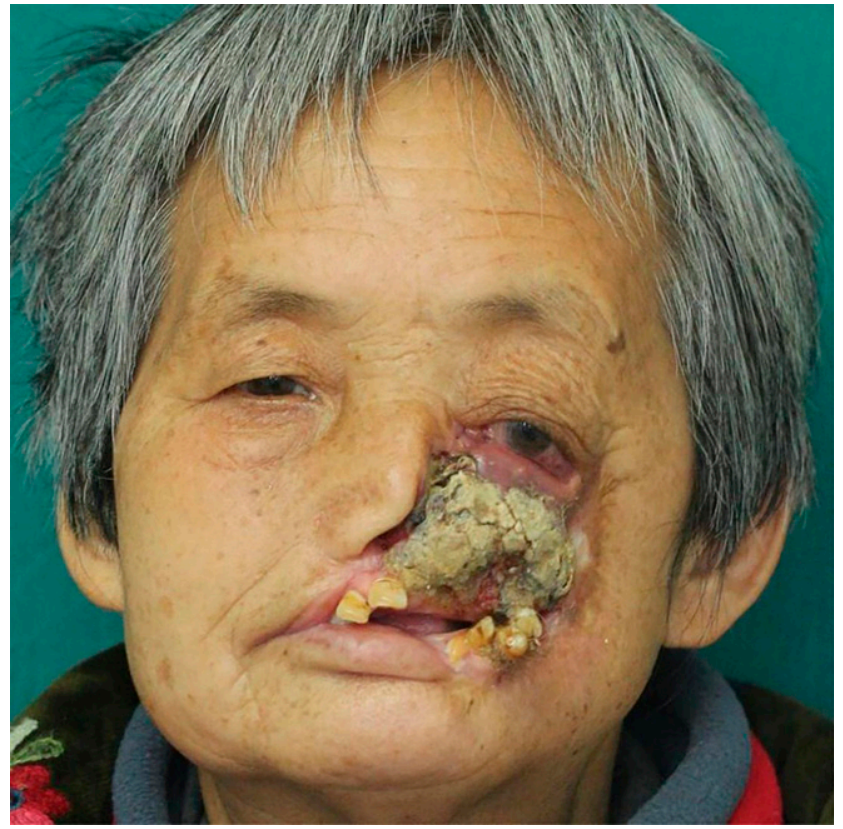

Figure 1. Preoperative photograph of noma with malignant transformation. This figure appears in color at www.ajtmh.org.

her condition was stable. However, because of economic problems, this patient and her family members refused.

\section{DISCUSSION}

Noma is an opportunistic infectious disease, which results from a pathogenic microorganism infection. It mainly occurs in poor countries in sub-Saharan Africa. Only sporadic reports are found in China. ${ }^{4-6}$ Noma often starts in the oral cavity and

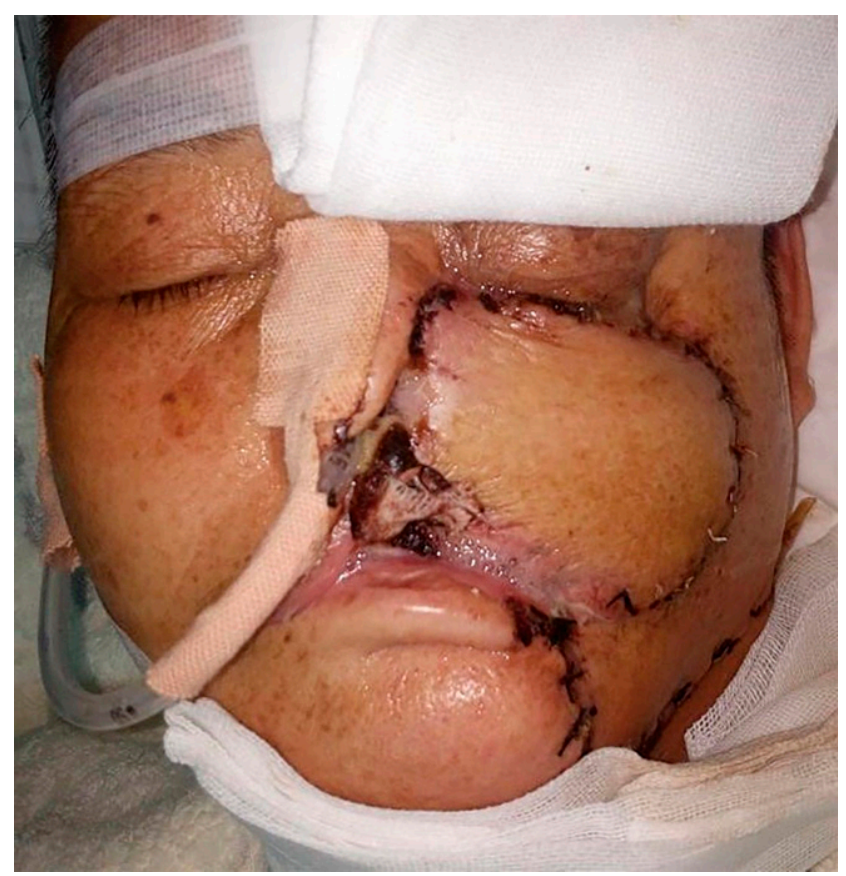

FIGURE 2. Three days after the operation. This figure appears in color at www.ajtmh.org.

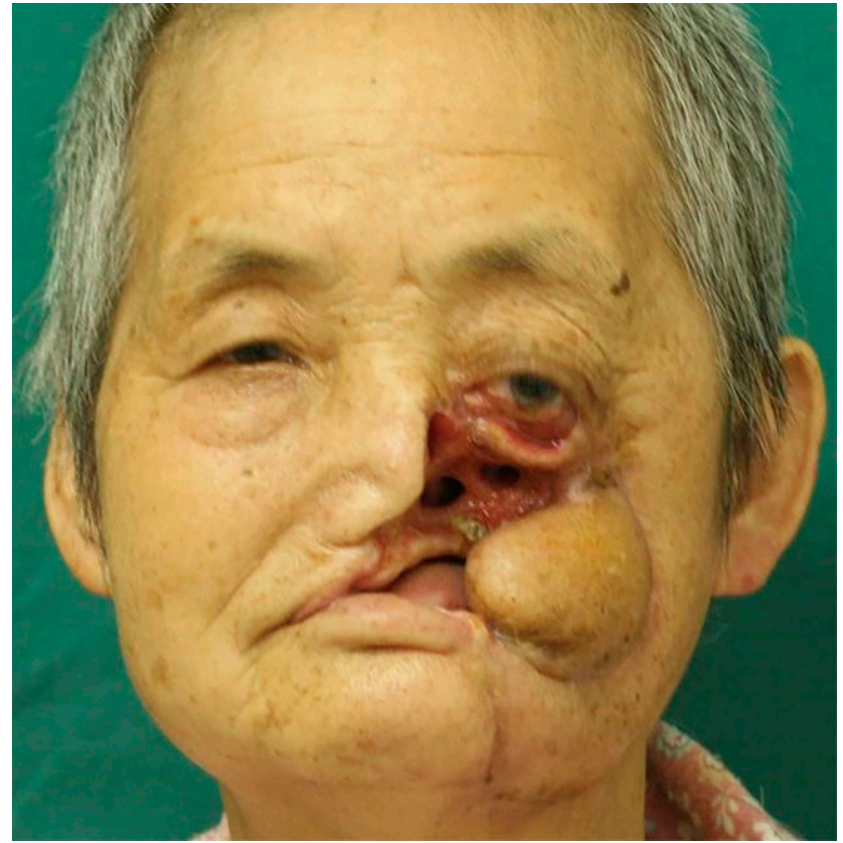

FIGURE 3. Six months postoperation. This figure appears in color at www.ajtmh.org.

spreads rapidly to the maxillofacial region and results in irreversible facial damage. ${ }^{7-9}$ Antibiotics, such as penicillin, have successfully reduced mortality of patients from $85 \%$ to $15 \%{ }^{6,7}$ Noma is characterized by sudden onset and rapid development, which is mainly manifested in the edema of the cheek and/or gingiva, which quickly invades the whole oral mucosa. The tissue necrosis of the edematous area turns black, leading to soft tissue defect, bone tissue necrosis, and tooth loss. ${ }^{9,10}$ The occurrence of infection further accelerates the progress of the disease, and systemic symptoms can include high fever, salivation, anorexia, diarrhea, crying, and irritability. $^{11}$

In the acute and necrotic stages, the rational use of antibiotics is important (the WHO suggests that penicillin and metronidazole should be the first choice). ${ }^{8,9}$ After the patient's necrotic tissue has shed itself, appropriate debridement can be performed. ${ }^{12}$ Noma's surgical reconstruction must rely on the basic principles of congenital, traumatic, and tumorous deformity repair and focus on the safe and reliable procedures of low-resource allocation. ${ }^{13,14}$

In this report, the patient suffered from measles 50 years ago before developing noma and she was unable to be treated because of limited personal economic resources. After a detailed assessment of the patient's condition, our treatment group tried to use the forearm flap to repair the patient's left facial defect. However, the flap dehiscence occurred 1 week after the operation, which resulted in unsatisfactory repair results. We consulted the relevant literature and found that Bouman and other scholars used various plastic operations to reconstruct the facial defects of 63 African patients with noma sequelae. The early complications were as high as $64 \%$, and the early success rate was only $59 \%$. It indirectly indicates that the more complicated the surgical procedure, the higher is the incidence of postoperative complications. ${ }^{15} \mathrm{We}$ also found that no noma-induced tumor has been previously reported. 
Many surgeons and stomatologists have rare chance to meet the patients with noma. When patients with noma sequelae are admitted, we suggest that a pathological biopsy of the diseased tissue be performed before operation to determine whether there is cancer. And the medical workers should develop a more standardized surgical treatment program depending on preoperative biopsy results. This case warns us that if the deformity changes from static to active and erodes the surrounding normal tissues, it should be treated on time to prevent malignant transformation of the affected tissue and improve the quality of life of the patients through safe and reliable repair and reconstruction.

Received December 5, 2019. Accepted for publication July 6, 2020.

Published online August 10, 2020.

Acknowledgment: We thank the Key Project Foundation of Anhui Province, China (No. KJ2016A480).

Authors' addresses: Xiang Ding, Department of Stomatology, The Second Affiliated Hospital of Bengbu Medical College, Bengbu, Anhui Province, China, E-mail: 18297301575@163.com. Qian-qian Wang and Jin-cheng Xu, Department of Stomatology, The First Affiliated Hospital of Bengbu Medical College, Bengbu, Anhui Province, China, E-mails: 1962580034@qq.com and w18895637183@163.com. Yang Zhou, Editorial Department of Journal of Bengbu Medical College, Bengbu, Anhui Province, China, E-mail: 364063948@qq.com.

\section{REFERENCES}

1. Feller L, Khammissa RAG, Altini M, Lemmer J, 2019. Noma (cancrum oris): an unresolved global challenge. Periodontol 2000 80: 189-199.
2. Jin LY, Ou XR, He ZJ, Xie XL, 2010. Maxillofacial deformity caused by cancrum oris: a case report. Hua Xi Kou Qiang Yi Xue Za Zhi 28: 342-344.

3. Song LX, Wu LY, Chang CK, Li X, 2010. Acute leukemia complicated with noma during induced remission: report of a case and review of literature. J Diagn Concepts Pract 9: 79-81.

4. Cheng J, Jiang L, 2017. Necrotizing stomatitis: a case report. Beijing J Stomatol 25: 169-170.

5. Srour ML, Marck K, Baratti-Mayer D, 2017. Noma: overview of a neglected disease and human rights violation. Am J Trop Med Hyg 96: 268-274.

6. Enwonwu CO, 2006. Noma--the ulcer of extreme poverty. N Engl $J$ Med 354: 221-224.

7. Leila Srour M, Marck KW, Baratti-Mayer D, 2015. Noma: neglected, forgotten and a human rights issue. Int Health 7: 149-150.

8. Enwonwu CO, 1995. Noma: a neglected scourge of children in sub-Saharan Africa. Bull World Health Organ 73: 541-545.

9. Feller L, Altini M, Chandran R, Khammissa RA, Masipa JN, Mohamed A, Lemmer J, 2014. Noma (cancrum oris) in the South African context. J Oral Pathol Med 43: 1-6.

10. Baratti-Mayer D, Pittet B, Montandon D, Bolivar I, Bornand JE, Hugonnet S, Jaquinet A, Schrenzel J, Pittet D; Geneva Study Group on Noma, 2003. Noma: an "infectious" disease of unknown aetiology. Lancet Infect Dis 3: 419-431.

11. Li BQ, 2006. Diseases of Oral Mucosa, 2nd edition. Beijing, China: People's Medical Publishing House, 36.

12. Weledji EP, Njong S, 2016. Cancrum oris (noma): the role of nutrition in management. J Am Coll Clin Wound Spec 7: 50-52.

13. Montandon $D, 2018$. The progress on noma disease and its surgical treatment. J Craniofac Surg 29: 539-542.

14. Shaye DA, Winters R, Rabbels J, Adentunji AS, Magee A, Vo D, 2019. Noma surgery. Laryngoscope 129: 96-99.

15. Bouman MA, Marck KW, Griep JE, Marck RE, Huijing MA, Werker PM, 2010. Early outcome of noma surgery. J Plast Reconstr Aesthet Surg 63: 2052-2056. 